Reply to : Sentinel node biopsy after neoadjuvant chemotherapy for breast cancer in patients with pre-treatment node-positive: Recommendation to optimize the performance

Simons, J. M.

2020-01

Simons , J M , Maaskant-Braat , A J G , Luiten , E J T , Leidenius , M H , van Nijnatten , T A , Boelens , P G , Koppert , L B , van der Pol , C C , van de Velde , C J H , Audisio, R A \& Smidt, M L 2020 , ' Reply to : Sentinel node biopsy after neoadjuvant chemotherapy for breast cancer in patients with pre-treatment node-positive: Recommendation to optimize the performance ' , European Journal of Surgical Oncology , vol. 46 , no. 1 , pp. 218-219 . https://doi.org/10.1016/j.ejso.2

http://hdl.handle.net/10138/325563

https://doi.org/10.1016/j.ejso.2019.10.022

publishedVersion

Downloaded from Helda, University of Helsinki institutional repository.

This is an electronic reprint of the original article.

This reprint may differ from the original in pagination and typographic detail.

Please cite the original version. 


\section{Reply to: Sentinel node biopsy after neoadjuvant chemotherapy for breast cancer in patients with pre-treatment node-positive: Recommendation to optimize the performance}

Keywords:

Axillary lymph node dissection

Axillary staging

Breast cancer

Neoadjuvant systemic therapy

Node-positive

Treatment-response
We appreciate the opportunity to provide a reply to the letter by G. Franceschini. In his letter, some advices to optimize the performance of sentinel lymph node biopsy (SLNB) are proposed for clinically node positive $(\mathrm{cN}+)$ patients treated with neoadjuvant chemotherapy (NAC). When a $\mathrm{cN}+$ patient achieves a pathologic complete response (pCR) of the axilla after NAC, a completion ALND may not be necessary. The main issue is the lack of an accurate less invasive staging procedure to safely replace ALND to identify such patients.

We agree with Franceschini that removing SLNs together with a marked node seems crucial to accurate staging of the axilla. Our group performed a systemic review and meta-analysis to evaluate the diagnostic accuracy of currently available less invasive staging procedures [1]. In this review, we compared the diagnostic accuracy of SLNB, MARI and of procedures in which SLNB and MARI are combined (i.e. excision of SLNs and a marked node). We found the following:

- 17 studies on the diagnostic accuracy of SLNB: overall false negative rate (FNR) of $17 \%$ and negative predictive value (NPV) ranging from $57 \%$ to $86 \%$

- 1 study on MARI: FNR 7\% and NPV 83\%

- 2 studies on a combination procedure (like Targeted Axillary Dissection): FNR 2-4\% and NPV 92-97\%

SLNB has been studied extensively compared to MARI or the combination procedure. Although our systematic review shows that a combination procedure (in which SLNs and a marked node are removed) appears most accurate, the evidence to support this is very limited. A similar procedure is being validated in the prospective multicenter Dutch RISAS trial [2]. Regarding SLNB, the

DOIs of original article: https://doi.org/10.1016/j.ejso.2019.10.020, https://doi.org/ 10.1016/j.ejso.2019.08.012. overall FNR appears to be fairly high and the NPV never exceeds $86 \%$. This means that residual axillary disease is missed in 1 out of 6 patients with a negative SLNB. This is highly undesirable, since positive lymph nodes found after NAC indicate therapy resistant disease. In case such disease is missed, patients may not receive appropriate adjuvant treatment. Accurate staging is therefore not only important to prevent overtreatment in patients with a pCR, but also to provide adjuvant regional treatment in case of residual disease. More research is needed to further shape patient-tailored axillary management for $\mathrm{cN}+$ patients treated with NAC, with de-escalation and escalation of treatment when necessary.

\section{References}

[1] Simons JM, et al. Diagnostic accuracy of different surgical procedures for axillary staging after neoadjuvant systemic therapy in node-positive breast cancer: a systematic review and meta-analysis. Ann Surg 2019;269:432-42.

[2] van Nijnatten TJA, et al. A novel less-invasive approach for axillary staging after neoadjuvant chemotherapy in patients with axillary node-positive breast cancer by combining radioactive iodine seed localization in the axilla with the sentinel node procedure (RISAS): a Dutch prospective multicenter validation study. Clin Breast Canc 2017;17:399-402.

J.M. Simons

Erasmus Medical Center Rotterdam, Department of Surgical Oncology, Rotterdam, the Netherlands

University Medical Center Utrecht, Department of Surgical Oncology, Utrecht, the Netherlands

A.J.G. Maaskant-Braat Maxima Medisch Centrum, Department of Surgical Oncology, Eindhoven, the Netherlands

E.J.T. Luiten Amphia Hospital, Department of Surgical Oncology, Breda, the Netherlands

M.H. Leidenius Helsinki University Hospital, Department of Breast Surgery, Helsinki, Finland

T.J.A. van Nijnatten

Maastricht University Medical Center+, Department of Radiology and Nuclear Medicine, Maastricht, the Netherlands

P.G. Boelens

Leiden University Medical Center, Department of Surgery, Leiden, the Netherlands 
L.B. Koppert

Erasmus Medical Center Rotterdam, Department of Surgical Oncology, Rotterdam, the Netherlands

C.C. van der Pol

Alrijne Hospital, Department of Surgical Oncology, Leiderdorp, the Netherlands

C.J.H. van de Velde Leiden University Medical Center, Department of Surgery, Leiden, the Netherlands

R.A. Audisio Institute of Clinical Sciences, Sahlgrenska University Hospital, Department of Surgery, Göteborg, Sweden
M.L. Smidt

Maastricht University Medical Center+, Department of Surgical Oncology, Maastricht, the Netherlands

Maastricht University Medical Center+, GROW - School for Oncology and Developmental Biology, Maastricht, the Netherlands

* Corresponding author. Department of Surgery, University Medical Center Utrecht, P.O. Box 85500, 3508, GA Utrecht, the Netherlands. E-mail address: j.m.simons@umcutrecht.nl (J.M. Simons).

14 October 2019

Available online 22 October 2019 